\title{
Synthesis of Multitopic Verdazyl Radical Ligands. Paramagnetic Supramolecular Synthons
}

\author{
Robin G. Hicks*, Bryan D. Koivisto, and Martin T. Lemaire
}

\section{Supporting Information}

General synthetic procedures. Unless stated otherwise, all reactions and manipulations were carried out under an argon atmosphere using standard Schlenk line techniques. The following solvents were dried and distilled under argon prior to use $\left(\mathrm{CH}_{2} \mathrm{Cl}_{2}, \mathrm{CH}_{3} \mathrm{CN}\right.$, and toluene, from $\mathrm{CaH}_{2}$; $\mathrm{DMF}$, from Alumina/CuSO 4 ; benzene, diethyl ether, and THF, from sodium/benzophenone). Carbonic acid 2,4dimethylcarbohydrazide $\mathbf{1 0}$ was prepared according to published procedures. ${ }^{1}$ All other reagents were obtained commercially and used as received, except where stated otherwise. ${ }^{1} \mathrm{H} /{ }^{13} \mathrm{C}$ NMR spectra were recorded on a $300 \mathrm{MHz}$ instrument except as otherwise stated. FT-IR spectra were recorded as $\mathrm{KBr}$ pressed discs on a FT-IR spectrometer $\left(4000-400 \mathrm{~cm}^{-1}\right)$. EPR spectra were recorded in fluid solution $\left(\mathrm{CH}_{2} \mathrm{Cl}_{2}\right.$ or toluene) on a $\mathrm{CW}$ spectrometer. Elemental analyses were carried out by Canadian Microanalytical Services Ltd., Vancouver, BC, Canada. Mass spectra were collected using electron impact (EI), or liquid secondary ion (LSIMS) techniques. Melting points are uncorrected. 


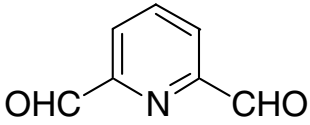

13

2,6-pyridinedicarboxaldehyde (13). A solution of anhydrous DMSO (13.2 g, 0.169 mol) in $\mathrm{CH}_{2} \mathrm{Cl}_{2}(18 \mathrm{~mL})$ was added slowly dropwise to a mechanically-stirred solution of oxalyl chloride $(7.86 \mathrm{~g}, 61.9 \mathrm{mmol})$ in $\mathrm{CH}_{2} \mathrm{Cl}_{2}(90 \mathrm{~mL})$ (gas evolution noted) at $-78^{\circ} \mathrm{C}$ (the temperature of the reaction was maintained at $-78^{\circ} \mathrm{C}$ until after the addition of $\mathrm{NEt}_{3}$ ). After the addition was complete, the solution was stirred for $5 \mathrm{~min}$, and then a solution of 2,6pyridinedimethanol (12) $(3.00 \mathrm{~g}, 0.0216 \mathrm{~mol})$ in anhydrous DMSO $(12 \mathrm{~mL})$ and $\mathrm{CH}_{2} \mathrm{Cl}_{2}(18 \mathrm{~mL})$ was added dropwise over a period of $15 \mathrm{~min}$. The solution was stirred for $20 \mathrm{~min}$, during which time the formation of a white precipitate was noted. Anhydrous $\mathrm{NEt}_{3}(\sim 30 \mathrm{~mL})$ was then slowly added dropwise to the reaction mixture. The reaction was let warm to room temperature, stirred for $2 \mathrm{~h}$, and then poured into $\sim 150 \mathrm{~mL}$ of water. The layers were separated and the aqueous layer was extracted with $3 \times 100 \mathrm{~mL}$ of $\mathrm{CH}_{2} \mathrm{Cl}_{2}$. The combined organic extracts were washed with $3 \times 100 \mathrm{~mL}$ of brine, dried over $\mathrm{MgSO}_{4}$, and filtered. The solvent was then removed under reduced pressure, and the tan solid was purified by vacuum sublimation at $\sim 55^{\circ} \mathrm{C}$ affording colorless crystals of 13, yield $2.5 \mathrm{~g}(86 \%)$. ${ }^{1} \mathrm{H}$ NMR (DMSO-d 6 ): $\delta 10.14(\mathrm{~s}, 2 \mathrm{H}), 8.16(\mathrm{~d}, 2 \mathrm{H}, J=7$ $\mathrm{Hz}), 8.06(\mathrm{t}, 1 \mathrm{H}, J=8 \mathrm{~Hz}) \mathrm{ppm} .{ }^{13} \mathrm{C}$ NMR $\left(\mathrm{CDCl}_{3}\right): \delta 192,153,138,125 \mathrm{ppm}$. FT-IR (KBr): $3083(\mathrm{~m}), 3052$ (w sh), 3015 (w), 2862 (m), 1719 (s), 1693 (m sh), 1597 (w), 1349 (s), 1299 (w), 1260 (s), 1208 (s), 1162 (w), 1085 (m), 992 (s), 912 (s), 892 (w sh), 805 (s), 785 (s sh), 723 (w), 698 (s), 622 (s), 491 (w) cm ${ }^{-1}$. Anal. Calcd for $\mathrm{C}_{7} \mathrm{H}_{5} \mathrm{NO}_{2}$ : C, 62.22; H, 3.73; N, 10.37. Found: C, 62.04; H, 3.74; N, 10.35. MS (CI methane): $\mathrm{m} / z 136$ $\left\{(\mathrm{MH})^{+}, 100 \%\right\}$.

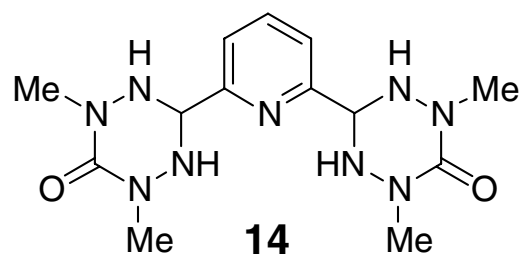

\section{2,6-pyridinebis(1,5-dimethyl-1,2,4,5-tetrazane-6-oxide-3-yl) (14). A} solution of freshly vacuum-sublimed 13 (1.00 g, $7.40 \mathrm{mmol})$ in $\mathrm{MeOH}$ (200 $\mathrm{mL})$ was added slowly dropwise to a solution of $\mathbf{1 0}(2.00 \mathrm{~g}, 16.9 \mathrm{mmol})$ in warm $\mathrm{MeOH}(40 \mathrm{~mL})$. After the addition was complete $(2.5 \mathrm{~h})$, the solution was refluxed for $18 \mathrm{~h}$. The solvent was then removed under reduced pressure and EtOAc $(\sim 20 \mathrm{~mL})$ was added to the solid residue, and the slurry was filtered. The solid was washed with cold EtOAc affording a pure white powder of 14, yield $2.2 \mathrm{~g} \mathrm{(89 \% ).} \mathrm{The} \mathrm{powder} \mathrm{was} \mathrm{recrystallized} \mathrm{by} \mathrm{slow}$ evaporation of a $\mathrm{MeOH} / \mathrm{EtOAc}$ mixture (approximately 1:10) at room temperature, yielding large transparent prisms of 14. ${ }^{1} \mathrm{H}$ NMR (DMSO-d $\left.)_{6}\right): \delta 7.88(\mathrm{t}, 1 \mathrm{H}, J=8 \mathrm{~Hz}), 7.55(\mathrm{~d}, 2 \mathrm{H}, J=8 \mathrm{~Hz}), 5.88(\mathrm{~d}, 4 \mathrm{H}, J=11 \mathrm{~Hz})$, $4.85(\mathrm{t}, 2 \mathrm{H}, J=11 \mathrm{~Hz}), 3.00(\mathrm{~s}, 12 \mathrm{H}) \mathrm{ppm} .{ }^{13} \mathrm{C} \mathrm{NMR}\left(\mathrm{CDCl}_{3}\right): \delta 155,154,139,124,69,38 \mathrm{ppm}$. FT-IR $(\mathrm{KBr})$ : 3481 (m br), 3402 (w br sh), 3247 (m), 3198 (m), 3070 (w), 2927 (w), 2874 (w sh), 1613 (s br), 1576 (s sh), 1500 (m sh), 1472 (m sh), 1432 (s), 1386 (s), 1307 (m), 1290 (w sh), 1222 (w), 1149 (m sh), 1123 (s), 1085 (m), 1023 (w), 1017 (w), 1000 (m), 962 (s), 886 (s), 869 (m sh), 805 (s), 753 (s), 729 (s), 685 (m), 668 (w sh), 654 (s), $628(\mathrm{~m}), 588(\mathrm{w}), 531$ (s br), $461(\mathrm{w}) \mathrm{cm}^{-1}$. Anal. Calcd for $\mathrm{C}_{13} \mathrm{H}_{21} \mathrm{~N}_{9} \mathrm{O}_{2}$ : C, 46.28; H, 6.31; N, 37.59. 
Found: C, 46.29; H, 6.40; N, 37.40. MS (LSIMS): $m / z 336\left\{(\mathrm{MH})^{+}, 100 \%\right\}, 290\left\{\left(\mathrm{M}-3 \mathrm{CH}_{3}\right)^{+}, 20\right\}$. Mp 201$203^{\circ} \mathrm{C}$.<smiles>CN1N=C(c2cccc(C3=NN(C)C(=O)N(C)N3C)n2)N(C)N(C)C1=O</smiles>

2,6-pyridinebis(1,5-dimethyl-6-oxoverdazyl-3-yl) (5). To a vigorously stirred solution of $\mathbf{1 4}(330 \mathrm{mg}, 0.98 \mathrm{mmol})$ in distilled water $(5 \mathrm{~mL})$ was added quickly dropwise (via a pasteur pipette) an aqueous solution $(7 \mathrm{~mL})$ of $\mathrm{NaIO}_{4}(650 \mathrm{mg}, 3.04 \mathrm{mmol})$ resulting in the precipitation of an intensely redcolored solid (gas-evolution noted). Stirring was continued for $25 \mathrm{~min}$ and then the reaction mixture was cooled in an ice bath for $30 \mathrm{~min}$. The red product was isolated by vacuum filtration, washed with $\sim 30 \mathrm{~mL}$ of cold distilled water, and air dried, yield $275 \mathrm{mg}$ (83\%). Chromatography of the red solid results in decomposition of the radical, as well, in the solid state is not long lived enough to get an adequate elemental analysis. FT-IR (KBr): 2946 (w), 2879 (w sh), 1685 (s), 1581 (m sh), 1573 (m), 1521 (w), 1462 (m), 1397 (w), 1344 (w), 1277 (m), 1194 (w), 1067 (w sh), 1044 (w), 990 (w), 821 (m), 736 (w), 717 (m), $692(\mathrm{w}), 650(\mathrm{~m}), 540(\mathrm{~m}) . \quad \lambda_{\max }\left(\mathrm{CH}_{2} \mathrm{Cl}_{2}\right)\left(\varepsilon\left(\mathrm{M}^{-1} \mathrm{~cm}^{-1}\right)\right): 409 \mathrm{~nm}\left(2.7 \times 10^{3}\right)$. High-res MS (EI): $\mathrm{m} / z$ 329.1344, Calcd for $\mathrm{C}_{13} \mathrm{H}_{15} \mathrm{~N}_{9} \mathrm{O}_{2} 329.1351$. Mp $90^{\circ} \mathrm{C}$ (dec.).

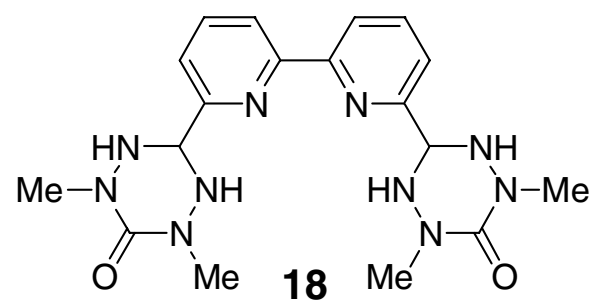

\section{2,2'-bipyridine-6,6'-bis(1,5-dimethyl-1,2,4,5-tetrazane-6-oxide-3-yl)}

(18). To a solution of warm 10 (990 mg, $8.38 \mathrm{mmol})$ in $\mathrm{MeOH}(20 \mathrm{~mL})$ was added slowly dropwise a fine slurry of 2,2'-bipyridine-6,6'dicarboxaldehyde 17 (880 mg, $4.15 \mathrm{mmol})$ in $\mathrm{MeOH}(250 \mathrm{~mL})$. After the addition was complete $(3 \mathrm{~h})$, the reaction mixture was refluxed for 18

h. The solvent was removed under reduced pressure affording a crude white solid. The solid was washed with $\mathrm{MeOH}$ to give pure 18, yield $1.55 \mathrm{~g}(90 \%)$. The crude solid was recrystallized by dissolution in hot $\mathrm{MeOH}$, filtering off the insolubles and cooling the filtrate in ice. ${ }^{1} \mathrm{H}$ NMR $\left(\mathrm{CD}_{3} \mathrm{OD}\right): \delta 8.54(\mathrm{~d}, 2 \mathrm{H}, J=8 \mathrm{~Hz}), 7.99$ (t, $2 \mathrm{H}, J=8 \mathrm{~Hz}), 7.61(\mathrm{~d}, 2 \mathrm{H}, J=7 \mathrm{~Hz}), 4.99(\mathrm{~s}, 2 \mathrm{H}), 3.07(\mathrm{~s}, 12 \mathrm{H}) \mathrm{ppm} .{ }^{13} \mathrm{C} \mathrm{NMR}\left(\mathrm{CD}_{3} \mathrm{OD}\right): \delta 157,156,155$, 140, 125, 123, 71, 38 ppm. FT-IR (KBr): 3424 (m, br), 3190 (m sh), 3168 (m), 3059 (w), 2934 (m), 2871 (w sh), 1613 (s), 1570 (m), 1516 (m), 1479 (w), 1439 (s), 1394 (s), 1357 (w), 1320 (w), 1203 (w), 1149 (m), 1129 (w sh), 1104 (m), 1078 (w sh), 993 (w), 973 (s), 913 (w), 893 (m), 876 (w sh), 799 (s), 751 (m), 723 (m), 686 (w), $649(\mathrm{~m}), 629(\mathrm{w}), 529(\mathrm{w}) \mathrm{cm}^{-1}$. Three attempts to obtain adequate elemental analysis data indicated the presence of $1 \mathrm{~mol}$ of $\mathrm{H}_{2} \mathrm{O}$ per mol of 18, Anal. Calcd for $\mathrm{C}_{18} \mathrm{H}_{24} \mathrm{~N}_{10} \mathrm{O}_{2} \bullet \mathrm{H}_{2} \mathrm{O}$ : C, 50.22; H, 6.09; N, 32.54 . Found: C, 49.98; H, 5.74; N, 32.20. HR MS (LSIMS): $m / z 413.2154\left\{(\mathrm{MH})^{+}, 100 \%\right\}$, Calcd for $\mathrm{C}_{18} \mathrm{H}_{24} \mathrm{~N}_{10} \mathrm{O}_{2}$

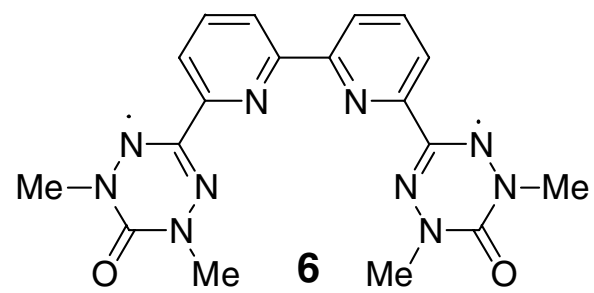
413.2165. $\mathrm{Mp} 194^{\circ} \mathrm{C}(\mathrm{dec}$.$) .$ 
2,2'-bipyridine-6,6'-bis(1,5-dimethyl-6-oxoverdazyl-3-yl) (6). A vigorously stirred slurry of 9 (200 mg, 0.48 $\mathrm{mmol})$ in DMF $(8 \mathrm{~mL})$ is heated until complete dissolution of the solid is noted, at which time a solution of $\mathrm{K}_{3} \mathrm{Fe}(\mathrm{CN})_{6}(990 \mathrm{mg}, 3.01 \mathrm{mmol})$ in aqueous $\mathrm{Na}_{2} \mathrm{CO}_{3}$ solution $(6 \mathrm{~mL}, 0.5 \mathrm{M})$ is added by pipette in large portions resulting in a color change to orange and the precipitation of a white solid. Distilled water $(\sim 10 \mathrm{~mL})$ is added to the mixture to dissolve the white precipitate, leaving a deep red precipitate. The solution is stirred for $\sim 20 \mathrm{~min}$ and then cooled in an ice bath. The red solid is collected by vacuum filtration, washed with cold distilled water $(\sim 30 \mathrm{~mL})$, and air dried affording 6, yield $180 \mathrm{mg}(91 \%)$. Attempts to recrystallize or chromatograph 6 result in decomposition of the radical. FT-IR (KBr): 2934 (w), 2871 (w sh), 1675 (s), 1576 (w sh), 1564 (m), 1453 (m), 1405 (m), 1391 (w sh), 1297 (w), 1263 (m), 1152 (w), 1106 (w), 1081 (w), 1024 (w), 990 (w), 808 (m), 742 (w), $717(\mathrm{~m}), 674(\mathrm{~m}), 632(\mathrm{w}), 546(\mathrm{~m}), 535(\mathrm{w} \mathrm{sh}) . \quad \lambda_{\max }\left(\mathrm{CH}_{2} \mathrm{Cl}_{2}\right)\left(\varepsilon\left(\mathrm{M}^{-1} \mathrm{~cm}^{-1}\right)\right)$ : 408nm $\left(2.2 \times 10^{3}\right)$. High-res MS (LSIMS): $\quad m / z 407.1686\left\{(\mathrm{MH})^{+}, 100 \%\right\}$, Calcd for $\mathrm{C}_{18} \mathrm{H}_{18} \mathrm{~N}_{10} \mathrm{O}_{2}$ 407.1695. Mp 130-140 ${ }^{\circ} \mathrm{C}$ (gradually decomposes over the temperature range).

6-bromopyridine-2-carbaldehyde ${ }^{3,4}$ (19) - $n$-BuLi (1.6M in hexanes, 26.6ml) was diluted<smiles>O=Cc1cccc(Br)n1</smiles>

19 by THF $(50 \mathrm{ml})$ and cooled to $-75^{\circ} \mathrm{C}$. To this stirring mixture, a solution of dibromopyridine $15(10 \mathrm{~g}, 42.2 \mathrm{mmol})$ in THF $(35 \mathrm{ml})$ was added dropwise, maintaining a temperature at/or below $-70^{\circ} \mathrm{C}(\sim 1.5$ hours $)$. Once the dropwise addition was complete the resulting dark green solution $\left(-70^{\circ} \mathrm{C}\right)$ was stirred for $30 \mathrm{~min}$. Then over a $30 \mathrm{sec}$. period, excess anhydrous DMF (4.9ml, 63.3mmol) was added. Upon the electrophile addition the reaction temperature elevated to $-40^{\circ} \mathrm{C}$, and the dark green solution became a dark turquoise colour. The mixture was then cooled to $-70^{\circ} \mathrm{C}$, and the acetone dry ice bath was removed and the reaction warmed to $0^{\circ} \mathrm{C}$. The reaction was then quenched with $\mathrm{MeOH}(30 \mathrm{ml})$, resulting in a yellow transparent solution. The reaction mixture was then extracted from aqueous saturated $\mathrm{NaHCO}_{3}(100 \mathrm{ml})$ with $\mathrm{CH}_{2} \mathrm{Cl}_{2}(3 \times 75 \mathrm{ml})$. The organic fractions were combined and the solvent was removed under reduced pressure. The crude aldehyde was purified using column chromatography ( silica, $\mathrm{CH}_{2} \mathrm{Cl}_{2}, \mathrm{R}_{\mathrm{f}}=0.50$ ) yielding $7.4 \mathrm{~g}(94.2 \%)$ of the aldehyde product (pure by TLC and $\left.{ }^{1} \mathrm{H}-\mathrm{NMR}\right)$. For crystalline material the product can be recrystallized from hexane. $\mathrm{mp} 80-81^{\circ} \mathrm{C} .{ }^{1} \mathrm{H} \mathrm{NMR}\left(\mathrm{CDCl}_{3}\right): \delta 9.97(\mathrm{~s}, 1 \mathrm{H}), 7.19(\mathrm{dd}, J=6.5,1.8 \mathrm{~Hz}, 1 \mathrm{H}), 7.76-$ $7.65(\mathrm{~m}, 2 \mathrm{H}) .{ }^{13} \mathrm{C} \mathrm{NMR}\left(\mathrm{CDCl}_{3}\right): \delta 191.8,153.7,142.7,139.6,132.9,120.5$. FT-IR (KBr): $3040(\mathrm{w}), 2872$ (w), 1731 (s), 1574 (m), 1554 (m), 1435 (s), 1412 (m), 1351 (w), 1219 (m), 1212 (m), 1163 (w), 1118 (s), 986 (m), $856(\mathrm{~m}), 796(\mathrm{~s}), 708(\mathrm{~m}), 633(\mathrm{~m}), \mathrm{cm}^{-1}$. Anal. Calcd for $\mathrm{C}_{6} \mathrm{H}_{4} \mathrm{BrNO}$ : C, 38.74; H, 2.17; N, 7.53. Found: C, 38.89; H, 2.19; N, 7.51. $\lambda_{\max }(\mathrm{MeOH})\left(\varepsilon\left(\mathrm{M}^{-1} \mathrm{~cm}^{-1}\right)\right): 268 \mathrm{~nm}\left(6.7 \times 10^{3}\right) . \mathrm{MS}(\mathrm{LSIMS}): \mathrm{m} / z, 186,188\left\{(\mathrm{MH})^{+}\right.$, 100\%). HR-MS: $184.9474+/-0.0003$ (184.9476 for $\left.\mathrm{M}^{+} \mathrm{C}_{6} \mathrm{H}_{4} \mathrm{BrNO}\right)$.

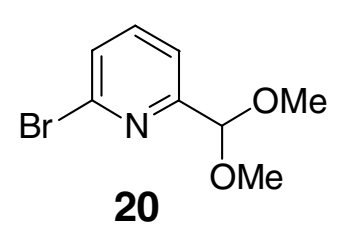

2-bromo-6-(dimethoxymethyl) pyridine ${ }^{5}$ (20) - 19 (15.0g, $\left.80.6 \mathrm{mmol}\right)$ was combined with $\mathrm{MeOH}(150 \mathrm{ml}$, sieve dried), a catalytic amount of para-toluylsulphonic acid hydrate 
(300mg) and trimethyl orthoformate $(35 \mathrm{ml}, 322.0 \mathrm{mmol})$ resulting in a yellow solution. This mixture was then refluxed for 4 hours resulting in a dark green/blue solution. Cooling the mixture to room temperature and removing most of the solvent under reduced pressure resulted in a crude liquid product. The product is distilled under reduced pressure (bp. $\left.85^{\circ} \mathrm{C} @ 1.5 \mathrm{~mm}\right)$ to yield a clear colorless liquid, 18.0g (96.0\%). ${ }^{1} \mathrm{H} \mathrm{NMR}\left(\mathrm{CDCl}_{3}\right)$ : $\delta 7.55(\mathrm{t}, \mathrm{J}=7.6 \mathrm{~Hz}, 1 \mathrm{H}), 7.47(\mathrm{~d}, J=7.6 \mathrm{~Hz}, 1 \mathrm{H}), 7.41(\mathrm{~d}, J=7.6 \mathrm{~Hz}, 1 \mathrm{H}), 5.25(\mathrm{~s}, 1 \mathrm{H}), 3.36(\mathrm{~s}, 6 \mathrm{H}) .{ }^{13} \mathrm{C} \mathrm{NMR}$ $\left(\mathrm{CDCl}_{3}\right): \delta$ 158.9, 141.5, 139.0, 128.1, 120.1, 103.7, 54.1. FT-IR (Neat): 3078 (w), 2993 (m), 2935 (s), 2830 (s), 1581 (s), 1557 (s), 1437 (s), 1408 (s), 1335 (s), 1271 (w), 1194 (s), 1155 (s), 1123 (br s), 1061 (br s), 982 (s), 921 (m), 880 (w), 795 (s), 750 (m), 712 (m), 653 (w), 638 (w), 618 (w), cm ${ }^{-1}$. Anal. Calcd for $\mathrm{C}_{8} \mathrm{H}_{10} \mathrm{BrNO}_{2}$ : C, 41.40; H, 4.34; N, 6.04. Found: C, 41.01; H, 4.20; N, 5.82. $\lambda_{\max }(\mathrm{MeOH})\left(\varepsilon\left(\mathrm{M}^{-1} \mathrm{~cm}^{-1}\right)\right): 268 \mathrm{~nm}\left(5.5 \times 10^{3}\right) . \mathrm{MS}$ (LSIMS): $m / z$ 201, 157, $233\left\{,(\mathrm{MH})^{+}, 100 \%\right\}$. HR-MS: $230.9892+/-0.0006\left(230.9895\right.$ for $\left.\mathrm{M}^{+} \mathrm{C}_{8} \mathrm{H}_{10} \mathrm{BrNO}_{2}\right)$.

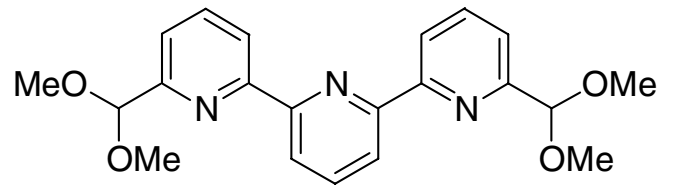

21
6,6"'-Bis-dimethoxymethyl-[2,2';6',2'] terpyridine $^{6}(21)-n$-BuLi $(1.6 \mathrm{M}$ in hexanes, $15.3 \mathrm{ml}, 24.6 \mathrm{mmol})$ was solubilized in THF (25ml) and cooled, to $-75^{\circ} \mathrm{C}$. To this a solution of $20(5.7 \mathrm{~g}$, 24.6mmol) in THF (15ml) was added dropwise, maintaining a temperature at/or below $-70^{\circ} \mathrm{C}$. Once the dropwise addition was

complete the resulting dark red solution was stirred for $30 \mathrm{~min}$. Then over a $20 \mathrm{~min}$ a cold $\left(0^{\circ} \mathrm{C}\right)$ solution of zinc chloride $\left(1.0 \mathrm{M}\right.$ in $\left.\mathrm{Et}_{2} \mathrm{O}, 27 \mathrm{ml}\right)$ was added maintaining a temperature at/or below $-70^{\circ} \mathrm{C}$. After the addition was complete the reaction was warmed to $0^{\circ} \mathrm{C}$, and $\mathrm{Pd}\left(\mathrm{PPh}_{3}\right)_{4}(0.57 \mathrm{~g}, 2 \mathrm{~mol} \%)$ and dibromopyridine $15(2.21 \mathrm{~g}$, $9.3 \mathrm{mmol}$ ) were added. The reaction mixture was then stirred for $30 \mathrm{~min}$, followed by refluxing under argon for $\sim 20 \mathrm{hrs}$ resulting in a large quantity of white precipitate. After leaving the mixture in a freezer overnight, the cold mixture was filtered and washed with cold THF. The precipitate was then basified in an aqueous sat. $\mathrm{NaHCO}_{3}$ solution, and extracted vigorously from a saturated EDTA solution using DCM. The organic fractions were then dried over $\mathrm{MgSO}_{4}$, and the solvent removed under reduced pressure yielding $2.8 \mathrm{~g}$ of the desired product $(78.6 \%) . \mathrm{mp} 82-84^{\circ} \mathrm{C} .{ }^{1} \mathrm{H}$ NMR $\left(\mathrm{CDCl}_{3}\right): \delta 8.56(\mathrm{~d}, J=7.5 \mathrm{~Hz}, 2 \mathrm{H}), 8.50(\mathrm{~d}, J=7.5 \mathrm{~Hz}, 2 \mathrm{H}), 7.90(\mathrm{t}, J$ $=7.5 \mathrm{~Hz}, 1 \mathrm{H}), 7.87(\mathrm{t}, J=7.5 \mathrm{~Hz}, 2 \mathrm{H}), 7.56(\mathrm{~d}, J=7.5 \mathrm{~Hz}, 2 \mathrm{H}) .{ }^{13} \mathrm{C} \mathrm{NMR}\left(\mathrm{CDCl}_{3}\right): \delta 156.9,155.7,155.3$, 137.9, 137.6, 121.5, 121.2, 121.0, 104.8, 54.1. FT-IR (KBr): 2994 (m), 2936 (m), 2890 (m), 2833 (m), 1567 (s), 1434 (s), 1357 (m), 1338 (s), 1254 (m), 1197 (s), 1114 (s), 1059 (br. s), 984 (s), 921 (m), 830 (m), 790 (s), 746 (m), 697 (m), 633 (s) $\mathrm{cm}^{-1}$. Anal. Calcd for $\mathrm{C}_{21} \mathrm{H}_{23} \mathrm{~N}_{3} \mathrm{O}_{4}$ : C, 66.13; H, 6.08; N, 11.02. Found: C, 65.81; $\mathrm{H}$, $6.10 ; \mathrm{N}, 10.91 . \lambda_{\max }\left(\mathrm{CHCl}_{3}\right)\left(\varepsilon\left(\mathrm{M}^{-1} \mathrm{~cm}^{-1}\right)\right): 283 \mathrm{~nm}\left(4.3 \times 10^{4}\right)$. MS (LSIMS): $m / z 382\left\{(\mathrm{MH})^{+}, 100 \%\right\}, 350\{(\mathrm{M}-$ $\left.\mathrm{CH}_{3} \mathrm{O}\right)^{+}, 90 \%$ \}. HR-MS: $382.1767+/-0.0012$ (382.1767 for $\mathrm{MH}^{+} \mathrm{C}_{21} \mathrm{H}_{24} \mathrm{~N}_{3} \mathrm{O}_{4}$ ).<smiles>O=Cc1cccc(-c2cccc(-c3cccc(C=O)n3)n2)n1</smiles>

$\left[2,2^{\prime} ; 6^{\prime}, 2^{\prime \prime}\right]$ Terpyridine-6,6"'-dicarbaldehyde ${ }^{7-10} \quad$ (22) - Acetic acid $(15 \mathrm{ml})$ and water $(25 \mathrm{ml})$ were combined with $21(2.0 \mathrm{~g}, 5.2 \mathrm{mmol})$ resulting 
in a milky white mixture. This mixture was stirred vigorously and refluxed for $1 \mathrm{hr}$. After cooling to room temperature the solution was evaporated to near dryness, and (neutralized) extracted from a sat. $\mathrm{NaHCO}_{3}$ solution using $\mathrm{CHCl}_{3}(3 \times 50 \mathrm{ml})$. The organic extracts were combined, dried over $\mathrm{MgSO}_{4}$, and the solvent removed under reduced pressure to yield $1.4 \mathrm{~g}$ of the product $(92.3 \%)$. Higher purity can be achieved by recrystallizing from $\mathrm{CHCl}_{3} . \mathrm{mp} 231-232^{\circ} \mathrm{C}$ (decomp. $234^{\circ} \mathrm{C}$ ). ${ }^{1} \mathrm{H} \mathrm{NMR}$ [500MHz] $\left(\mathrm{CDCl}_{3}\right): \delta 10.19(\mathrm{~s}, 2 \mathrm{H}), 8.84$ $(\mathrm{dd}, J=7.5,1.4 \mathrm{~Hz}, 2 \mathrm{H}), 8.65(\mathrm{~d}, J=7.52 \mathrm{H}), 8.08-7.99(\mathrm{~m}, 5 \mathrm{H}) .{ }^{13} \mathrm{C} \mathrm{NMR}\left(\mathrm{CDCl}_{3}\right): \delta 193.9,156.7,154.7$, 152.6, 138.5, 138.2, 125.38, 122.1, 121.8. FT-IR (KBr): 3079 (m), 2855 (m), 1722 (s), 1584 (s), 1575 (s), 1563 (m), 1460 (m), 1451 (m), 1435 (s), 1386 (w), 1347 (m), 1284 (w), 1257 (m), 1218 (m), 1151 (w), 1110 (m), 1074 (m), $992(\mathrm{~m}), 922(\mathrm{w}), 867$ (m), $792(\mathrm{~s}), 728(\mathrm{~m}), 671(\mathrm{~m}), 633(\mathrm{~m}) \mathrm{cm}^{-1}$. Anal. Calcd for $\mathrm{C}_{17} \mathrm{H}_{11} \mathrm{~N}_{3} \mathrm{O}_{2}$ : C, 70.58 ; $\mathrm{H}, 3.83 ; \mathrm{N}, 14.53$. Found: C, 70.35; H, 3.77; N, 15.32. $\lambda_{\max }\left(\mathrm{CHCl}_{3}\right)\left(\varepsilon\left(\mathrm{M}^{-1} \mathrm{~cm}^{-1}\right)\right): 292 \mathrm{~nm}\left(4.2 \times 10^{4}\right) . \mathrm{MS}(\mathrm{EI})$ : $m / z, 289\left\{(\mathrm{M})^{+}, 100 \%\right\}, 289\left\{(\mathrm{M}-\mathrm{CHO})^{+}, 60 \%\right\}$. HR-MS: $290.0931+/-0.0002\left(290.0930\right.$ for $\left.\mathrm{MH}^{+} \mathrm{C}_{17} \mathrm{H}_{12} \mathrm{~N}_{3} \mathrm{O}_{2}\right)$.<smiles>CN1NC(c2cccc(-c3cccc(-c4cccc(C5NN(C)C(=O)N(C)N5)n4)n3)n2)NN(C)C1=O</smiles>

\section{[2,2';6',2']Terpyridine-6,6"-bis(1,5-dimethyl-1,2,4,5-} tetrazane-6-oxide-3-yl) (23) - $\mathbf{1 0}(1.02 \mathrm{~g}, 8.6 \mathrm{mmol})$ was solubilized in $15 \mathrm{ml}$ of $\mathrm{MeOH} / \mathrm{CHCl}_{3}(1: 1)$ and the solution was gently refluxed. To this gently refluxing solution $\mathbf{2 2}$ $(1.25 \mathrm{~g}, 4.32 \mathrm{mmol})$ in $\mathrm{CHCl}_{3} / \mathrm{MeOH}(1: 1)(175 \mathrm{ml})$ was

added dropwise over 3 hrs using a pressure equalizing dropping funnel. After the addition was complete the reaction was gently refluxed overnight (10 hrs). The solution was then cooled and solvent removed to yield a white/yellow solid. The product was triturated using hot ethyl acetate, yielding $1.7 \mathrm{~g} \mathrm{(80.4 \% ).} \mathrm{mp} \mathrm{199-201}{ }^{\circ} \mathrm{C}$ (decomp). ${ }^{1} \mathrm{H}$ NMR (DMSO): $\delta 8.72(\mathrm{~d}, J=8 \mathrm{~Hz}, 2 \mathrm{H}), 8.61$ (d, $\left.J=8 \mathrm{~Hz}, 2 \mathrm{H}\right), 8.14$ (t, $\left.J=8 \mathrm{~Hz}, 1 \mathrm{H}\right), 8.05$ (t, $J$ $=8 \mathrm{~Hz}, 2 \mathrm{H}), 7.65(\mathrm{~d}, J=8 \mathrm{~Hz}, 2 \mathrm{H}), 5.83(\mathrm{~d}, J=10.5 \mathrm{~Hz}, 4 \mathrm{H}), 5.02(\mathrm{t}, J=10.5 \mathrm{~Hz}, 2 \mathrm{H}), 3.01(\mathrm{~s}, 12 \mathrm{H}) .{ }^{13} \mathrm{C}$ NMR (MeOH): $\delta 157.2,156.9,156.3,155.2,139.6,139.4,124.9,122.9,122.4,70.7,38.4$. FT-IR (KBr): 3421 (m), 3242 (m), 3073 (w), 2931 (m), 2871 (w), 1628 (s), 1569 (s), 1504 (m), 1434 (s), 1389 (m), 1312 (w), 1267 (w), 1241 (w), 1110 (br m), 1080 (m), 992 (w), 952 (w), 888 (w), 800 (m), 731 (w), 633 (w), $530(\mathrm{w}) \mathrm{cm}^{-1}$. Anal. Calcd for $\mathrm{C}_{23} \mathrm{H}_{27} \mathrm{~N}_{11} \mathrm{O}_{2}$ : C, 56.43; H, 5.56; N, 31.47. Found: C, 56.18; H, 5.76; N, 31.50. $\lambda_{\max }\left(\mathrm{CHCl}_{3}\right)$ $\left(\varepsilon\left(\mathrm{M}^{-1} \mathrm{~cm}^{-1}\right)\right): 290 \mathrm{~nm}\left(5.8 \times 10^{4}\right)$. MS (LSIMS): $\mathrm{m} / \mathrm{z} 490\left\{(\mathrm{MH})^{+}, 100 \%\right\}$. HR-MS: $490.2437+/-0.0010$ (490.2427for $\mathrm{MH}^{+} \mathrm{C}_{23} \mathrm{H}_{28} \mathrm{~N}_{11} \mathrm{O}_{2}$ ).

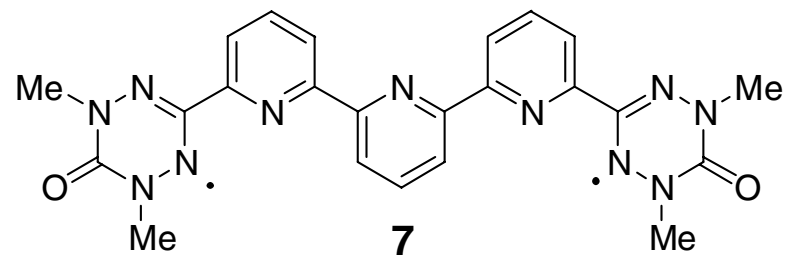

solution of $\mathrm{K}_{3} \mathrm{Fe}(\mathrm{CN})_{6}(0.61 \mathrm{~g}, 1.84 \mathrm{mmol})$ in aqueous $\mathrm{Na}_{2} \mathrm{CO}_{3}$ solution $(10 \mathrm{~mL}, 0.5 \mathrm{M})$ was added by pipette in large portions resulting in a color change to deep orange and the precipitation of a white solid occurred.

$\left[2,2^{\prime} ; 6^{\prime}, 2^{\prime \prime}\right]$ Terpyridine-6,6"'-bis(1,5-dimethyl-6oxoverdazyl-3-yl) (7) - A vigorously stirred slurry of $\mathbf{2 3}$ $(0.15 \mathrm{~g}, 0.31 \mathrm{mmol})$ in DMF $(8 \mathrm{~mL})$ is heated until complete dissolution of the solid was observed, at which time a 
Distilled water $(\sim 10 \mathrm{~mL})$ was added to the mixture to dissolve the white precipitate. The reaction was then left to stir for $30 \mathrm{~min}$. and then put in an ice bath for 30min after which time it was filtered and washed with water, followed by diethyl ether, yielding the diradical, 0.105g (70.9\%). mp $160^{\circ} \mathrm{C}$ (decomp). FT-IR (KBr): $3066(\mathrm{~m})$, 2938 (m), 1693 (s), 1563 (s), 1537 (s), 1460 (s), 1370 (s), 1258 (m), 1173 (w), 1152 (w), 1124 (w), 1078 (w), 1027 (w), 993 (w), 820 (m), 763 (m), 737 (w), 701 (m) 638 (m), 540 (m) cm . Anal. Calcd for $\mathrm{C}_{23} \mathrm{H}_{21} \mathrm{~N}_{11} \mathrm{O}_{2}$ : C, 57.14; H, 4.38; N, 31.87. Found: C, 56.30; H, 4.44; N, 31.06. $\lambda_{\max }\left(\mathrm{CHCl}_{3}\right)\left(\varepsilon\left(\mathrm{M}^{-1} \mathrm{~cm}^{-1}\right)\right): 297 \mathrm{~nm}\left(4.6 \times 10^{4}\right)$,

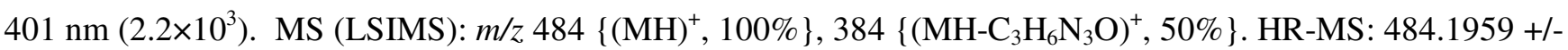
0.0010 (484.1958 for $\mathrm{MH}^{+} \mathrm{C}_{23} \mathrm{H}_{22} \mathrm{~N}_{11} \mathrm{O}_{2}$ ).

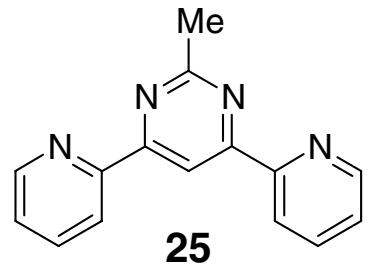

2-methyl-4,6-bis(pyridin-2'-yl)pyrimidine (25). Acetamidine hydrochloride $(1.7 \mathrm{~g}$, $0.018 \mathrm{~mol})$ was slurried in absolute $\mathrm{EtOH}(200 \mathrm{~mL})$ and $\mathrm{NaOH}$ pellets $(805 \mathrm{mg}, 0.020$ mol) were added until complete dissolution of all solid was noted (solution became cloudy). 24 (3.6 g, $0.017 \mathrm{~mol}$ ) was then added in portions over a period of $20 \mathrm{~min}$. Following the addition, the mixture was heated to reflux and a gradual color change from pale orange to peach and finally to dull dark yellow (the color at reflux) was observed with concomitant precipitation of a white solid $(\mathrm{NaCl})$. Refluxing was continued for $2 \mathrm{~h}$ and then the mixture was cooled to room temperature. Air was bubbled through the solution for about $10 \mathrm{~min}$, and the $\mathrm{pH}$ was lowered to neutral by addition of saturated ammonium chloride solution. All of the $\mathrm{EtOH}$ was removed under reduced pressure, affording a forest green aqueous solution containing a white precipitate. The flask was cooled in ice for $0.5 \mathrm{~h}$, and then the off-white solid was collected by vacuum filtration, washed with cold distilled water and dried. Chromatography (basic alumina, 4:1 hexanes/EtOAc) affords 25 as a fluffy white solid, yield $1.65 \mathrm{~g}(40 \%) .{ }^{1} \mathrm{H} \mathrm{NMR}\left(\mathrm{CDCl}_{3}\right): \delta 9.11$ (s, 1H), $8.75(\mathrm{~d}, 2 \mathrm{H}, J=6 \mathrm{~Hz}), 8.47(\mathrm{~d}, 2 \mathrm{H}, J=8 \mathrm{~Hz}), 7.84(\mathrm{dd}, 2 \mathrm{H}, J=8,2 \mathrm{~Hz}), 7.37(\mathrm{dd}, 2 \mathrm{H}, J=6,2 \mathrm{~Hz}), 2.88$ (s, 3H) ppm. ${ }^{13} \mathrm{C}$ NMR $\left(\mathrm{CDCl}_{3}\right): \delta 168,164,155,150,137,125,122,111,26$ ppm. FT-IR (KBr): $3105(\mathrm{vw})$, 3059 (vw), 3037 (w), 2997 (w), 2923 (vw), 1601 (m), 1580 (s), 1560 (s), 1541 (s), 1475 (m), 1434 (m), 1398 (m), 1371 (m), 1342 (w), 1287 (vw), 1266 (vw), 1250 (vw), 1229 (vw), 1187 (vw), 1163 (vw), 1146 (vw), 1118 (vw), 1094 (w), 1044 (w), 995 (m), 918 (w), 895 (vw), 837 (w), 799 (m), 755 (s), 741 (w sh), 734 (m), 652 (m), $620(\mathrm{~m}), 606(\mathrm{vw}), 582(\mathrm{w}), 558(\mathrm{vw}) \mathrm{cm}^{-1}$. Anal. Calcd for $\mathrm{C}_{15} \mathrm{H}_{12} \mathrm{~N}_{4}: \mathrm{C}, 72.56 ; \mathrm{H}, 4.87 ; \mathrm{N}, 22.57$. Found: C, 72.31; H, 4.75; N, 22.36. MS (CI methane): $m / z 249\left\{(\mathrm{MH})^{+}, 100 \%\right\}$. Mp 135-136 ${ }^{\circ}$.

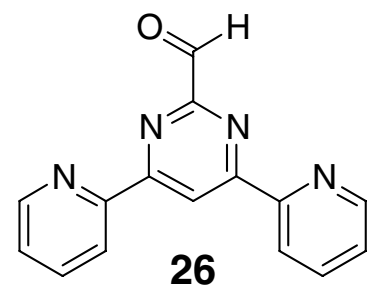

4,6-bis(pyridin-2'-yl)pyrimidine-2-carboxaldehyde (26). 25 (1.32 g, $5.3 \mathrm{mmol})$ and $\mathrm{SeO}_{2}(880 \mathrm{mg}, 8 \mathrm{mmol})$ were added to a 3-neck rbf containing argon-sparged dioxane $(50 \mathrm{~mL})$, and the mixture was heated to $90^{\circ} \mathrm{C}$ with stirring under an argon atmosphere. At this temperature, distilled water $(0.2 \mathrm{~mL})$ was added to the reaction mixture and the solution was subsequently heated to $130^{\circ} \mathrm{C}$ for $96 \mathrm{~h}$. The progress of the reaction was

monitored by ${ }^{1} \mathrm{H}$ NMR (DMSO- $\mathrm{d}_{6}$ ) until the disappearance of the methyl signal of $\mathbf{2 5}$ (may take longer than 96 
h) was noted. Over the course of the reaction a fine black solid precipitates out $\left(\mathrm{Se}^{\mathrm{o}}\right)$ and the solution darkens to a salmon color. The reaction was stopped when all, or most, of $\mathbf{2 5}$ was reacted and the reaction flask was subsequently cooled to room temperature. Distilled water $(50 \mathrm{~mL})$ was added and the flask was cooled in an ice bath, affording an off-white solid which was collected by gravity filtration. Trituration of the solid with $\mathrm{CH}_{2} \mathrm{Cl}_{2}$ $(3 \times 30 \mathrm{~mL})$ and evaporation of the $\mathrm{CH}_{2} \mathrm{Cl}_{2}$ extracts under reduced pressure affords $\mathbf{2 6}$ as a crude tan solid, which was recrystallized from $\mathrm{CH}_{2} \mathrm{Cl}_{2} / \mathrm{Et}_{2} \mathrm{O}(1: 3)$ at $-10^{\circ} \mathrm{C}$. More 26 can be recovered by evaporation of the dioxane/water filtrate. Combined yield, $400 \mathrm{mg}(28 \%) .{ }^{1} \mathrm{H} \mathrm{NMR}\left(\mathrm{CD}_{2} \mathrm{Cl}_{2}\right): \delta 10.25(\mathrm{~s}, 1 \mathrm{H}), 9.53(\mathrm{~s}, 1 \mathrm{H}), 8.80$ $(\mathrm{d}, 2 \mathrm{H}, J=4 \mathrm{~Hz}), 8.63(\mathrm{~d}, 2 \mathrm{H}, J=8 \mathrm{~Hz}), 7.92(\mathrm{dd}, 2 \mathrm{H}, J=8,2 \mathrm{~Hz}), 7.48(\mathrm{dd}, 2 \mathrm{H}, J=7,2 \mathrm{~Hz}) \mathrm{ppm} .{ }^{13} \mathrm{C} \mathrm{NMR}$ $\left(\mathrm{CD}_{2} \mathrm{Cl}_{2}\right): \delta 191,165,159,153,150,137,126,122,115$ ppm. FT-IR (KBr): 3099 (w), $3054(\mathrm{w}), 3008$ (w), 2934 (vw), 2843 (w), 1724 (s), 1593 (s sh), 1573 (s sh), 1559 (s), 1536 (m sh), 1516 (m), 1471 (m), 1419 (m), 1368 (m), 1291 (vw), 1263 (w), 1226 (w), 1155 (w), 1112 (w), 1087 (w), 1050 (w), 990 (m), 927 (m), 907 (w sh), 836 (w), 794 (m), 762 (s), 740 (s), 677 (w), 649 (s), 617 (m), 546 (w) cm-1. Anal. Calcd for $\mathrm{C}_{15} \mathrm{H}_{10} \mathrm{~N}_{4} \mathrm{O}: \mathrm{C}$, 68.69; H, 3.84; N, 21.36. Found: C, 67.29; H, 3.71; N, 20.91. MS (EI): $m / z 262\left(\mathrm{M}^{+}, 100 \%\right), 234$ \{(M-CO) ${ }^{+}$, 50\}, $156\left\{(\mathrm{M}-\mathrm{CO}-\mathrm{py})^{+}, 30\right\}$. High-res MS: m/z 262.0856, Calcd for $\mathrm{C}_{15} \mathrm{H}_{10} \mathrm{~N}_{4} \mathrm{O}$ 262.0856. Mp 186-188 ${ }^{\circ} \mathrm{C}$.

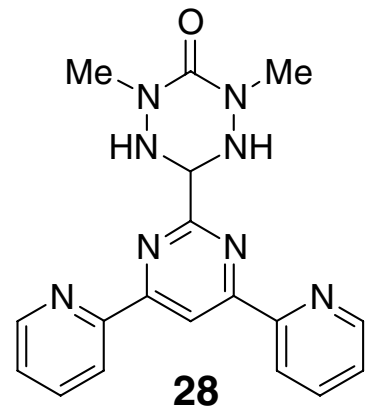

1,5-dimethyl-\{4,6-(dipyridin-2'-yl)pyrimidin-2'-yl\}-1,2,4,5-tetrazane-6-oxide (28). A solution of $26(520 \mathrm{mg}, 1.87 \mathrm{mmol})$ in $\mathrm{MeOH}(100 \mathrm{~mL})$ was added dropwise to a warm solution of $\mathbf{1 0}(250 \mathrm{mg}, 2.12 \mathrm{mmol})$ in $\mathrm{MeOH}(10 \mathrm{~mL})$. After the addition was complete $(1 \mathrm{~h})$, the solution was refluxed for $18 \mathrm{~h}$. The reaction mixture was then cooled to room temperature, and the solvent was removed under reduced pressure to yield an off-white powder that was washed with EtOAc $(10 \mathrm{~mL})$ to afford pure $\mathbf{2 8}$, yield $564 \mathrm{mg}$ (79\%). Compound 28 was recrystallized by dissolving into hot $\mathrm{MeOH}$, filtering the insolubles, and letting the saturated solution cool slowly to room temperature ${ }^{1} \mathrm{H}$ NMR (DMSO- $\left.{ }_{6}\right)$ : $\delta 9.29(\mathrm{~s}, 1 \mathrm{H}), 8.82(\mathrm{~d}, 2 \mathrm{H}, J=4 \mathrm{~Hz}), 8.68(\mathrm{~d}, 2 \mathrm{H}, J=7 \mathrm{~Hz}), 8.08(\mathrm{dd}, 2 \mathrm{H}, J=7,2 \mathrm{~Hz}), 7.64(\mathrm{dd}, 2 \mathrm{H}, J=6,2$ $\mathrm{Hz}), 5.98(\mathrm{~d}, 2 \mathrm{H}, J=10 \mathrm{~Hz}), 5.05(\mathrm{t}, 1 \mathrm{H}, J=10 \mathrm{~Hz}), 3.02(\mathrm{~s}, 6 \mathrm{H}) \mathrm{ppm} .{ }^{13} \mathrm{C} \mathrm{NMR}\left(\mathrm{CD}_{2} \mathrm{Cl}_{2}\right): \delta 164,163.8,155$, 153, 150, 138, 126, 122, 112, 71, 38 ppm. FT-IR (KBr): 3242 (m), 3105 (vw), 3054 (w), 2951 (w sh), 2911 (w), 2860 (w sh), 1630 (s), 1593 (m sh), 1576 (m), 1562 (m), 1530 (s), 1490 (m), 1471 (m), 1422 (s), 1371 (s), 1311 (m), 1266 (w), 1249 (vw), 1226 (w), 1141 (m sh), 1124 (m), 1087 (w), 1064 (vw), 1041 (vw), 993 (m), 950 (m), 899 (vw), 856 (m), 808 (w), 779 (s), 737 (m), 725 (m), 708 (vw), 660 (m sh), 649 (s), 617 (m), 552 (w), 512 (vw) $\mathrm{cm}^{-1}$. Anal. Calcd for $\mathrm{C}_{18} \mathrm{H}_{18} \mathrm{~N}_{8} \mathrm{O}: \mathrm{C}, 59.66 ; \mathrm{H}, 5.01 ; \mathrm{N}, 30.92$. Found: C, 59.53; H, 5.22; N, 30.22. MS<smiles>CN1N=C(c2nc(-c3ccccn3)cc(-c3ccccn3)n2)N(C)N(C)C1=O</smiles>
(LSIMS): $m / z 363\left\{(\mathrm{MH})^{+}, 100 \%\right\} . \mathrm{Mp} 180^{\circ} \mathrm{C}(\mathrm{dec}$.$) .$

1,5-dimethyl-3-\{4,6-bis(pyridin-2"-yl)pyrimidin-2'-yl\}-6-oxoverdazyl (8). To a slurry of 28 (200 mg, $0.55 \mathrm{mmol})$ in distilled water $(5 \mathrm{~mL})$ was added 35 drops of an 
aqueous $\mathrm{HCl}$ solution $(1 \mathrm{M})$ resulting in a clear yellow solution. $\mathrm{NaIO}_{4}(195 \mathrm{mg}, 0.91 \mathrm{mmol})$ was added to the above vigorously stirring solution in portions causing immediate precipitation of a microcrystalline dark purple solid. Stirring was continued for $10 \mathrm{~min}$ and then the reaction mixture was cooled in an ice bath for $30 \mathrm{~min}$. The product was isolated by vacuum filtration, washed with $\sim 30 \mathrm{~mL}$ of cold distilled water and air dried, yield 200 mg (95\%). FT-IR (KBr): 3504 (m br), 3373 (m br), 3094 (vw), 3054 (vw), 2940 (vw), 2700 (br w), 1684 (s), 1624 (w), 1579 (s), 1530 (s), 1473 (w), 1436 (w), 1414 (w), 1368 (w), 1308 (w), 1237 (m), 1169 (vw), 1118 (vw), 1087 (vw), 1033 (w), 996 (w), 967 (vw), 899 (vw), 839 (s), 805 (w), 777 (m), 742 (w), 700 (w), 674 (vw), $649(\mathrm{vw}), 617(\mathrm{w}), 541(\mathrm{w}), 521(\mathrm{vw}) \mathrm{cm}^{-1} . \lambda_{\max }\left(\mathrm{CH}_{2} \mathrm{Cl}_{2}\right)\left(\varepsilon\left(\mathrm{M}^{-1} \mathrm{~cm}^{-1}\right)\right): 402 \mathrm{~nm}\left(1.0 \times 10^{3}\right)$. High-res MS (EI): 359.1371 ( $\left.\mathrm{M}^{+}, 100 \%\right)$, Calcd for $\mathrm{C}_{18} \mathrm{H}_{15} \mathrm{~N}_{8} \mathrm{O} 359.1367$. Mp $155^{\circ} \mathrm{C}$ (dec.).

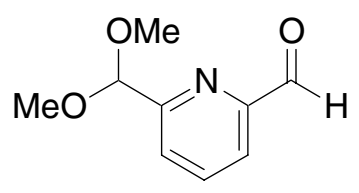

29

6-Dimethoxymethyl-pyridine-2-carbaldehyde (29) - $n$-BuLi (1.6M in hexanes, $27.0 \mathrm{ml})$ was diluted by THF $(50 \mathrm{ml})$ and cooled to $-75^{\circ} \mathrm{C}$. To this stirring mixture, a solution of $20(10 \mathrm{~g}, 43.1 \mathrm{mmol})$ in THF $(20 \mathrm{ml})$ was added drop wise, maintaining a temperature at/or below $-70^{\circ} \mathrm{C}(\sim 1.5$ hours $)$. Once the drop wise addition was complete the resulting dark red solution $\left(-70^{\circ} \mathrm{C}\right)$ was stirred for $30 \mathrm{~min}$. Then over a $30 \mathrm{sec}$. period, excess anhydrous DMF $(4.2 \mathrm{ml}, 53.9 \mathrm{mmol})$ was added. Upon the electrophile addition the reaction temperature elevated to $-40^{\circ} \mathrm{C}$, and the dark red solution darkened. The mixture was then cooled to $-70^{\circ} \mathrm{C}$, and the acetone dry ice bath was removed and the reaction warmed to $0^{\circ} \mathrm{C}$. The reaction was then quenched with $\mathrm{MeOH}(30 \mathrm{ml})$, resulting in a yellow transparent solution. The reaction mixture was then extracted from aqueous saturated $\mathrm{NaHCO}_{3}(100 \mathrm{ml})$ with $\mathrm{CH}_{2} \mathrm{Cl}_{2}(3 \times 75 \mathrm{ml})$. The organic fractions were combined and the solvent was removed under reduced pressure. The crude aldehyde was purified using fractional vacuum distillation $\left(47^{\circ} \mathrm{C}\right.$ at $\left.1.5 \mathrm{~mm}\right)$ yielding $6.8 \mathrm{~g}$ (87.1\%) of the product. ${ }^{1} \mathrm{H}$ NMR $\left(\mathrm{CDCl}_{3}\right): \delta 9.98(\mathrm{~s}, 1 \mathrm{H}), 7.82-7.79(\mathrm{~m}, 2 \mathrm{H}), 7.68(\mathrm{t}, J=4.5 \mathrm{~Hz}, 1 \mathrm{H}), 5.34(\mathrm{~s}$, 1H), $3.32(\mathrm{~s}, 6 \mathrm{H}) .{ }^{13} \mathrm{C} \mathrm{NMR}\left(\mathrm{CDCl}_{3}\right): \delta$ 193.2, 158.1, 152.1, 137.6, 125.5, 121.2, 103.6, 53.8. FT-IR (Neat): 2937 (m), 2832 (m), 1713 (s), 1590 (m), 1445 (m), 1342 (m), 1286 (w), 1257 (w), 1208 (m), 1193 (m), $1111(\mathrm{~s})$, 1062 (s, br), 990 (m), 836 (m), 795 (m), 744 (w), 699 (w), 631 (m) cm ${ }^{-1}$. Anal. Calcd for $\mathrm{C}_{9} \mathrm{H}_{11} \mathrm{NO}_{3}$ : C, 59.66; $\mathrm{H}, 6.12 ; \mathrm{N}, 7.73$. Found: $\mathrm{C}, 59.55 ; \mathrm{H}, 6.02 ; \mathrm{N}, 7.61 . \lambda_{\max }(\mathrm{MeOH})\left(\varepsilon\left(\mathrm{M}^{-1} \mathrm{~cm}^{-1}\right)\right): 268 \mathrm{~nm}\left(6.7 \times 10^{3}\right) . \mathrm{MS}$ (LSIMS): m/z $150\left\{(\mathrm{M}-\mathrm{OMe})^{+}, 100 \%\right\}, 152\left\{(\mathrm{M}-\mathrm{CHO})^{+}, 100 \%\right\}, 182\left\{(\mathrm{MH})^{+}, 100 \%\right\}$. HR-MS: $182.0822+/-$ 0.0005 (182.0817 for $\mathrm{MH}^{+} \mathrm{C}_{9} \mathrm{H}_{12} \mathrm{NO}_{3}$ ).

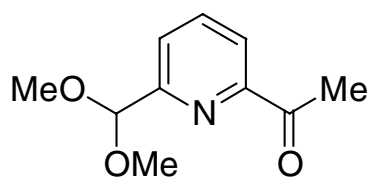

30

1-(6-Dimethoxymethyl-pyridin-2-yl)-ethanone (30) - $n$-BuLi (1.6M in hexanes, $27.0 \mathrm{ml})$ was diluted by THF $(70 \mathrm{ml})$ and cooled to $-75^{\circ} \mathrm{C}$. To this stirring mixture, a solution of $20(10 \mathrm{~g}, 43.1 \mathrm{mmol})$ in THF $(20 \mathrm{ml})$ was added drop wise, maintaining a temperature at/or below $-70^{\circ} \mathrm{C}(\sim 1.5$ hours $)$. Once the drop wise addition was complete the resulting dark red solution $\left(-70^{\circ} \mathrm{C}\right)$ was stirred for $30 \mathrm{~min}$. Then over a $30 \mathrm{sec}$. period, excess 
anhydrous dimethylacetamide (DMA) $(5.0 \mathrm{ml}, 53.9 \mathrm{mmol})$ was added. Upon the electrophile addition the reaction temperature elevated to $-40^{\circ} \mathrm{C}$, and the dark red solution darkened. The mixture was then cooled to $-70^{\circ} \mathrm{C}$, and the acetone dry ice bath was removed and the reaction warmed to $0^{\circ} \mathrm{C}$. The reaction was then quenched with $\mathrm{MeOH}(30 \mathrm{ml})$, resulting in a yellow transparent solution. The reaction mixture was then extracted from aqueous saturated $\mathrm{NaHCO}_{3}(100 \mathrm{ml})$ with $\mathrm{CH}_{2} \mathrm{Cl}_{2}(3 \times 75 \mathrm{ml})$. The organic fractions were combined and the solvent was removed under reduced pressure. The crude ketone was purified using fractional vacuum distillation $\left(71^{\circ} \mathrm{C}\right.$ at $1.5 \mathrm{~mm})$ yielding $5.7 \mathrm{~g}(67.8 \%)$ of the product. ${ }^{1} \mathrm{H} \mathrm{NMR}\left(\mathrm{CDCl}_{3}\right): \delta 7.90(\mathrm{dd}, \mathrm{J}=7.5,1.0 \mathrm{~Hz}, 1 \mathrm{H}), 7.78(\mathrm{t}, J=7.5$ $\mathrm{Hz}, 1 \mathrm{H}), 7.64(\mathrm{dd}, \mathrm{J}=7.5,1.0 \mathrm{~Hz}, 1 \mathrm{H}), 5.30(\mathrm{~s}, 1 \mathrm{H}), 3.36(\mathrm{~s}, 6 \mathrm{H}), 2.64(\mathrm{~s}, 3 \mathrm{H}) .{ }^{13} \mathrm{C} \mathrm{NMR}\left(\mathrm{CDCl}_{3}\right): \delta 200.1$, 157.1, 152.9, 137.5, 124.6, 121.4, 104.4, 54.1, 25.7. FT-IR (Neat): 2936 (m), 2831 (m), 1700 (s), 1586 (m), 1454 (m), 1420 (m), 1360 (s), 1293 (m), 1217 (m), 1193 (m), 1113 (s), 1062 (s, br), 987 (m), 959 (w), 928 (w), $817(\mathrm{~m}), 772(\mathrm{w}), 594(\mathrm{~m}) \mathrm{cm}^{-1}$. Anal. Calcd for $\mathrm{C}_{10} \mathrm{H}_{13} \mathrm{NO}_{3}$ : C, 61.53; H, 6.71; N, 7.18. Found: C, 61.91; H, 6.53; N, 7.11. $\lambda_{\max }(\mathrm{MeOH})\left(\varepsilon\left(\mathrm{M}^{-1} \mathrm{~cm}^{-1}\right)\right): 261 \mathrm{~nm}\left(8.1 \times 10^{3}\right)$. MS (LSIMS): $\mathrm{m} / z, 196\left\{(\mathrm{MH})^{+}, 50 \%\right\}, 164$ $\left\{(\mathrm{MH})^{+}, 100 \%\right\}$. HR-MS: $196.0970+/-0.0004$ (196.0973 for $\left.\mathrm{MH}^{+} \mathrm{C}_{10} \mathrm{H}_{13} \mathrm{NO}_{3}\right)$.

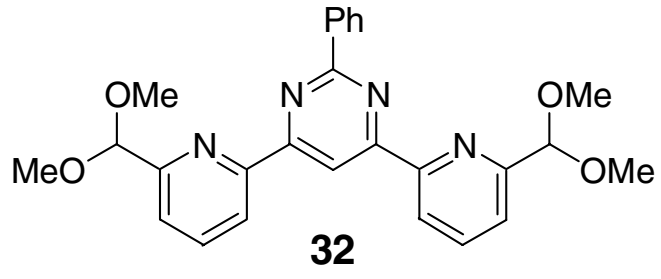

4,6-Bis-(6-dimethoxymethyl-pyridin-2-yl)-2-phenyl-pyrimidine (32) - 29 (2.05g $11.3 \mathrm{mmol})$ was vigorously suspended in an aqueous $\mathrm{KOH}(1.2 \mathrm{~g}, 21.5 \mathrm{mmol})$ solution $(75 \mathrm{ml})$. To this emulsion, a solution of $\mathbf{3 0}(2.1 \mathrm{~g}, 10.7 \mathrm{mmol})$ in $\mathrm{MeOH}(25 \mathrm{ml})$ was added slowly (over $3 \mathrm{hrs}$ ) via dropwise addition $\left(\right.$ at $\left.0^{\circ} \mathrm{C}\right)$. The resulting

enone obtained was yellow gum-like product, and after several attempts could not be isolated as pure material. The near solid product was then extracted from aqueous with methylene chloride $(3 \times 30 \mathrm{ml})$ and the organic fractions dried over $\mathrm{MgSO}_{4}$, and the solvent was removed under reduced pressure. The enone (yellow oil) was then solubilized in $\mathrm{MeOH}(150 \mathrm{ml})$ and combined with benzamidine hydrochloride $(1.68 \mathrm{~g}, 10.7 \mathrm{mmol})$, and $\mathrm{KOH}$ $(1.2 \mathrm{~g}, 21.5 \mathrm{mmol})$. The reaction was then allowed to stir at room temperature for $3 \mathrm{hrs}$, and then gently refluxed for 4 hrs. During the reflux air was bubbled through the mixture. After the reflux the yellow solution becomes a bright red/salmon coloured solution. The mixture was then cooled to $0^{\circ} \mathrm{C}$ in an icebath and the resulting precipitate collected, and washed with ice-cold methanol $(3 \times 20 \mathrm{ml})$ resulting in pure product $1.93 \mathrm{~g}(39.2 \%$ base on ketone) by ${ }^{1} \mathrm{H}-\mathrm{NMR}$. The product can be further purified by chromatography $\left(\mathrm{N}\right.$. alumina, $\left.\mathrm{CHCl}_{3}, \mathrm{R}_{\mathrm{f}}=0.6\right)$ or by recrystallization from hexane. mp $152-154^{\circ} \mathrm{C} .{ }^{1} \mathrm{H} \mathrm{NMR}\left(\mathrm{CDCl}_{3}\right): \delta 9.30(\mathrm{~s}, 1 \mathrm{H}) 8.70(\mathrm{~m}, 4 \mathrm{H}), 7.95(\mathrm{t}, J=7.7$ $\mathrm{Hz}, 2 \mathrm{H}), 7.69(\mathrm{~d}, \mathrm{~J}=7.7,2 \mathrm{H}), 7.53(\mathrm{~m}, 3 \mathrm{H}), 5.53(\mathrm{~s}, 2 \mathrm{H}), 3.49(\mathrm{~s}, 12 \mathrm{H}) .{ }^{13} \mathrm{C} \mathrm{NMR}\left(\mathrm{CDCl}_{3}\right): \delta 164.3,164.1$, 157.6, 154.3, 138.1, 137.8, 130.9, 128.7, 128.4, 122.8, 122.0, 112.2, 105.0, 54.3. FT-IR (KBr): 2936 (m), 2831 (m), 1700 (s), 1586 (m), 1454 (m), 1420 (m), 1360 (s), 1293 (m), 1217 (m), 1193 (m), 1113 (s), 1062 (s, br), 987 (m), 959 (w), 928 (w), 817 (m), 772 (w), 594 (m) $\mathrm{cm}^{-1}$. Anal. Calcd for $\mathrm{C}_{26} \mathrm{H}_{26} \mathrm{~N}_{4} \mathrm{O}_{4}$ : C, 68.11; H, 5.72; N, 12.22. Found: C, 67.72; H, 5.66; N, 12.23. $\lambda_{\max }\left(\mathrm{CHCl}_{3}\right)\left(\varepsilon\left(\mathrm{M}^{-1} \mathrm{~cm}^{-1}\right)\right): 272 \mathrm{~nm}\left(1.5 \times 10^{5}\right), 319 \mathrm{~nm}\left(4.8 \times 10^{3}\right)$. 


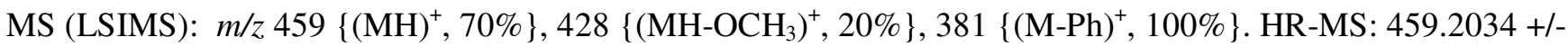
0.0013 (459.2032 for $\mathrm{MH}^{+} \mathrm{C}_{26} \mathrm{H}_{27} \mathrm{~N}_{4} \mathrm{O}_{4}$ ).<smiles>O=Cc1cccc(-c2cc(-c3cccc(C=O)n3)nc(-c3ccccc3)n2)n1</smiles>

6-[6-(6-formylpyridin-2-yl)-2-phenylpyrimidin-4-yl]-pyridine-2carbaldehyde (33) - Acetic acid (15ml) and water (25ml) was combined with $32(1.5 \mathrm{~g}, 3.3 \mathrm{mmol})$ resulting in a milky white mixture. This mixture was stirred vigorously and refluxed for $1 \mathrm{hr}$, over which time the mixture became transparent and slightly yellow in colour within minutes of becoming clear the solution precipitated a white precipitate. After cooling to room temperature the solution was evaporated to near dryness, and neutralized and extracted from a sat. $\mathrm{NaHCO}_{3}$ solution using $\mathrm{CHCl}_{3}(3 \times 50 \mathrm{ml})$. The organic extracts were combined dried over $\mathrm{MgSO}_{4}$, and the solvent removed under reduced pressure to yield $1.14 \mathrm{~g}$ of the product $(95.1 \%)$. Elemental purity can be achieved using column chromatography (N. Alumina/ $\mathrm{CHCl}_{3}-\mathrm{R}_{\mathrm{f}}=0.60$ ), or by recrystallizing from MeOH. mp 206-208 ${ }^{\circ} \mathrm{C} .{ }^{1} \mathrm{H}$ NMR $\left(\mathrm{CDCl}_{3}\right): \delta 10.28(\mathrm{~s}, 2 \mathrm{H}), 9.43(\mathrm{~s}, 1 \mathrm{H}), 8.97-8.91(\mathrm{~m}, 2 \mathrm{H}), 8.71-8.68(\mathrm{~m}$, $2 \mathrm{H}), 8.11-8.06(\mathrm{~m}, 4 \mathrm{H}), 7.58-7.53(\mathrm{~m}, 3 \mathrm{H}) .{ }^{13} \mathrm{C} \mathrm{NMR}\left(\mathrm{CDCl}_{3}\right): \delta 193.6,164.6,163.7,155.3,152.9,138.4$, 137.6, 131.3, 128.9, 128.6, 126.1, 123.1, 111.7. FT-IR (KBr): 2824 (w), 1715 (s), 1584 (m), 1561 (s), 1538 (s), 1460 (w), 1446 (m), 1372 (s), 1345 (m), 1221 (m), 1148 (w), 1072 (w), 993 (w), 895 (w), 815 (r S12 w), 760 (m), $706(\mathrm{~m}), 695(\mathrm{~m}), 654(\mathrm{~m}), 632(\mathrm{~m}) \mathrm{cm}^{-1}$. Anal. Calcd for $\mathrm{C}_{22} \mathrm{H}_{14} \mathrm{~N}_{4} \mathrm{O}_{2}$ : C, 72.12; H, 3.85; N, 15.29. Found: $\quad \mathrm{C}, 71.88 ; \mathrm{H}, 4.02 ; \mathrm{N}, 15.07 . \lambda_{\max }\left(\mathrm{CHCl}_{3}\right)\left(\varepsilon\left(\mathrm{M}^{-1} \mathrm{~cm}^{-1}\right)\right): 270 \mathrm{~nm}\left(3.3 \times 10^{4}\right), 322 \mathrm{~nm}\left(8.6 \times 10^{3}\right) . \mathrm{MS}$ (LSIMS): $m / z 367\left\{(\mathrm{MH})^{+}, 100 \%\right\}, 289\left\{(\mathrm{M}-\text { phenyl })^{+}, 30 \%\right\}$. HR-MS: $367.1200+/-0.0010\left(367.1195\right.$ for $\mathrm{MH}^{+}$ $\mathrm{C}_{22} \mathrm{H}_{15} \mathrm{~N}_{4} \mathrm{O}_{2}$ ).<smiles>CN1NC(c2cccc(-c3cc(-c4cccc(C5NN(C)C(=O)N(C)N5)n4)nc(-c4ccccc4)n3)n2)NN(C)C1=O</smiles>

(1:1) (175ml) and added dropwise over 5hrs using a pressure equalizing dropping funnel. After the addition was complete the reaction was gently refluxed overnight (10 hrs). The solution (yellow) was then cooled and solvent removed to yield a white/yellow solid. The product was triturated using hot ethyl acetate, yielding $0.99 \mathrm{~g}$ (85.3\%). mp 171-172 ${ }^{\circ} \mathrm{C}$ (decomp). ${ }^{1} \mathrm{H} \mathrm{NMR}\left(\mathrm{CDCl}_{3}\right): \delta 9.29(\mathrm{~s}, 1 \mathrm{H}), 8.76-8.66(\mathrm{~m}, 4 \mathrm{H}), 7.96(\mathrm{t}, \mathrm{J}=7.8 \mathrm{~Hz}, 2 \mathrm{H})$, 7.59-7.53 (m, 5H), 5.56 (br. s, 4H), 5.03 (s, 2H), 3.16 (s, 12H). ${ }^{13} \mathrm{C} \mathrm{NMR}\left(\mathrm{CDCl}_{3}\right): \delta 164.2,164.0,155.6,155.0$, 154.5, 138.4, 137.7, 131.1, 128.8, 128.5, 125.3, 122.0, 111.9, 69.7, 38.5. FT-IR (KBr): 3415 (m), 3229 (m), 2963 (w) 1627 (s), 1563 (s), 1538 (s), 1459 (m), 1448 (m), 1431 (m), 1373 (s), 1261 (m), 1095 (br m), 951 (m), 817 (m), 763 (w), 702 (w), 648 (m), 530 (w) cm $\mathrm{cm}^{-1}$. Anal. Calcd for $\mathrm{C}_{28} \mathrm{H}_{30} \mathrm{~N}_{12} \mathrm{O}_{2}$ : C, 59.35; H, 5.34; N, 29.66. Found: $\mathrm{C}, 59.42 ; \mathrm{H}, 5.46 ; \mathrm{N}, 29.71 . \lambda_{\max }\left(\mathrm{CHCl}_{3}\right)\left(\varepsilon\left(\mathrm{M}^{-1} \mathrm{~cm}^{-1}\right)\right): 273 \mathrm{~nm}\left(3.7 \times 10^{4}\right), 321 \mathrm{~nm}\left(1.2 \times 10^{4}\right) . \quad M S$ 


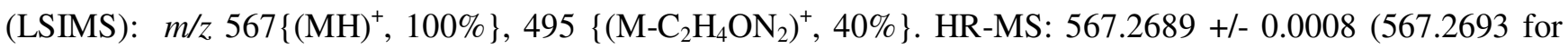
$\mathrm{MH}^{+} \mathrm{C}_{28} \mathrm{H}_{31} \mathrm{~N}_{12} \mathrm{O}_{2}$ ). 
<smiles>CN1N=C(c2cccc(-c3cc(-c4cccc(C5=NN(C)N(C)C(=O)N5C)n4)nc(-c4ccccc4)n3)n2)N(C)N(C)C1=O</smiles>

4,6-Bis-[6-(1,5-dimethyl-6-oxoverdazyl-3-yl)-pyridin-2yl]-2-phenyl-pyrimidine (9) - para-Benzoquinone ( $p \mathrm{BQ})$ $(0.12 \mathrm{~g}, 1.06 \mathrm{mmol})$ was added to a solution of $34(0.20 \mathrm{~g}$, $0.35 \mathrm{mmol})$ in $\mathrm{CHCl}_{3}(25 \mathrm{ml})$, and the yellow solution was refluxed for 25 minutes. The resulting deep red solution was cooled to room temperature, and the solvent removed under reduced pressure. The crude material was then triturated using hot ethyl acetate $(3 \times 10 \mathrm{ml})$, yielding the diradical, $0.135 \mathrm{~g}(68.2 \%) . \mathrm{mp} 161-166^{\circ} \mathrm{C}$ (decomposes over the range). FT-IR (KBr): 3253 (w), 3066 (w), 2938 (w), 1693 (s), 1563 (s), 1536 (s), 1460 (m), 1426 (m), 1369 (s), 1255 (m), 993 (w), 821 (m), 764 (w), 637 (m) cm ${ }^{-1}$. Anal. Calcd for $\mathrm{C}_{28} \mathrm{H}_{24} \mathrm{~N}_{12} \mathrm{O}_{2}$ : C, 59.99; H, 4.32; $\mathrm{N}, 29.98$. Found: $\mathrm{C}, 59.17 ; \mathrm{H}, 4.33 ; \mathrm{N}, 28.23 . \lambda_{\max }\left(\mathrm{CHCl}_{3}\right)\left(\varepsilon\left(\mathrm{M}^{-1} \mathrm{~cm}^{-1}\right)\right): 264 \mathrm{~nm}\left(4.1 \times 10^{4}\right), 318 \mathrm{~nm}\left(1.5 \times 10^{4}\right)$, $399 \mathrm{~nm}\left(2.3 \times 10^{3}\right)$. MS (LSIMS): $\mathrm{m} / z$ 560\{(M) $\left.{ }^{+}, 100 \%\right\}$. HR-MS: $560.2142+/-0.0005$ (566.2145 for $\mathrm{MH}^{+}$ $\mathrm{C}_{28} \mathrm{H}_{31} \mathrm{~N}_{12} \mathrm{O}_{2}$ ).

EPR Spectra of diradicals 7 and 9 and monoradical 8: All three spectra give rise to a complex splitting pattern that is typical of 1,5-dimethyl-6-oxoverdazyls regardless of the nature of the substituent at C3. The reasons for this have been discussed (see reference 15 in main text). The spectra can be simulated with the gvalue and hyperfine parameters below and suitable linewidth modifications. The fact that the spectra of the diradical species appear essentially the same as that of the monoradical is indicative of extremely weak/negligible exchange coupling between the two radical centers. This is not unexpected as the two radicals are separated by three aromatic groups and linked at their C3 centers which do not carry much spin density.

\begin{tabular}{ccccc}
\hline Compound & g-factor & $a\left(\mathrm{~N}_{1,5}\right)(\mathrm{G})$ & $a\left(\mathrm{~N}_{2,4}\right)(\mathrm{G})$ & $a\left(6 \mathrm{CH}_{3}\right)(\mathrm{G})$ \\
\hline 7 & 2.0039 & 6.5 & 5.3 & 5.3 \\
8 & 2.0037 & 6.5 & 5.3 & 5.3 \\
9 & 2.0034 & 6.3 & 5.4 & 5.3
\end{tabular}

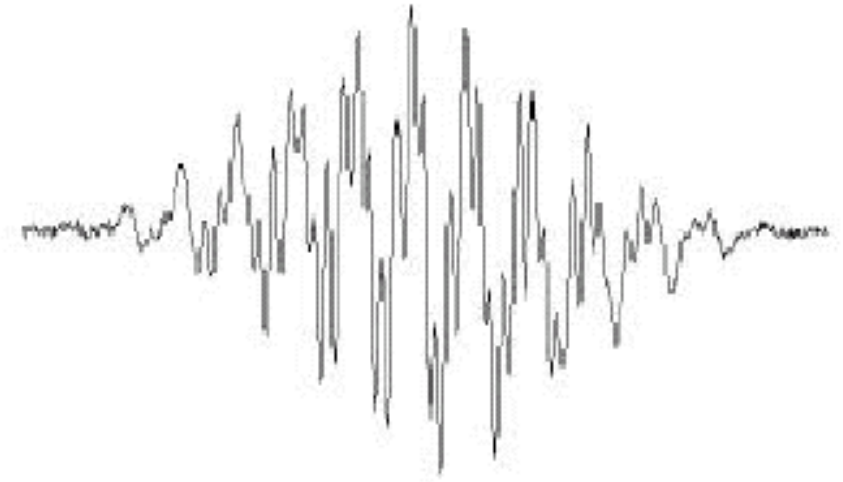

\section{EPR Spectrum for monoradical 8}

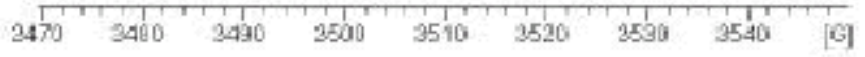




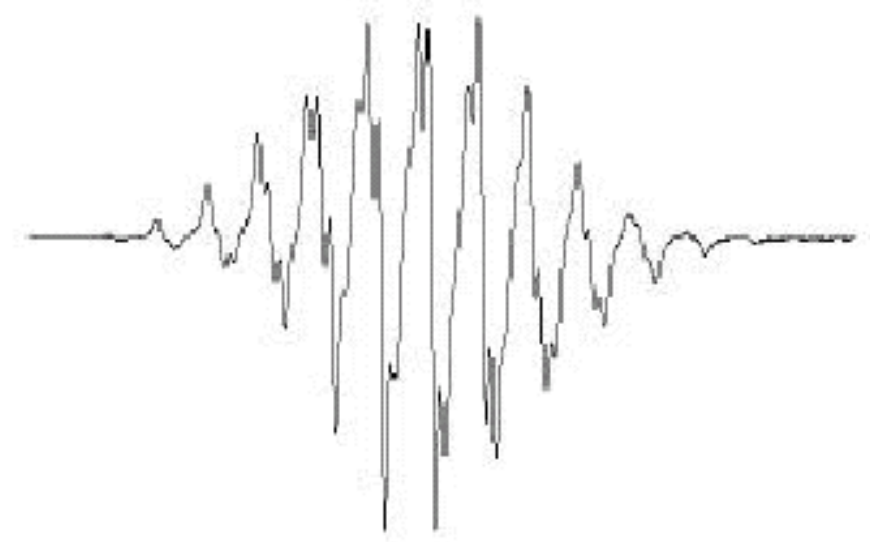

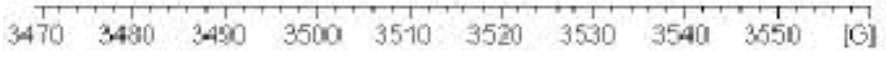

EPR Spectrum of diradical 9
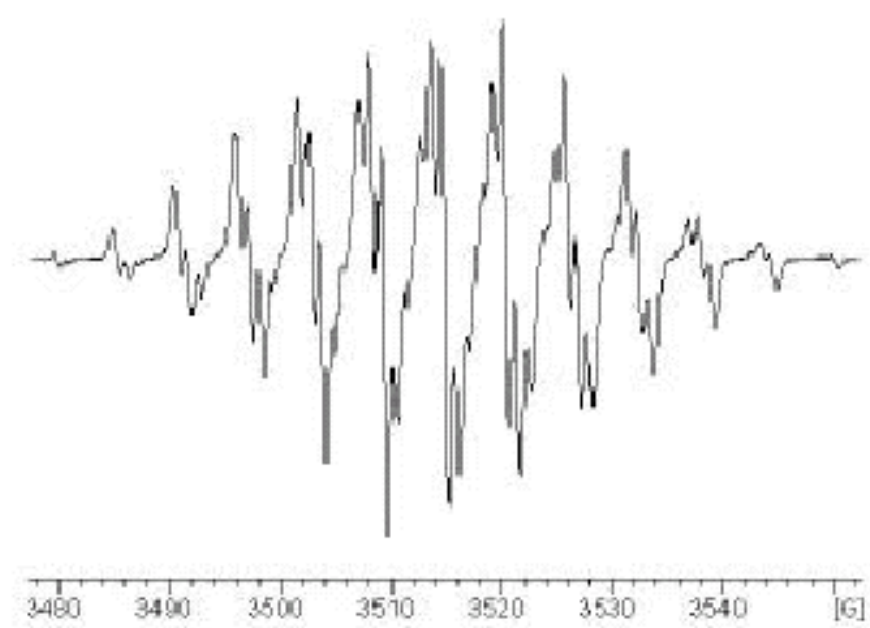

EPR Spectrum of diradical 7

\section{References}

(1) Barr, C. L.; Chase, P. A.; Hicks, R. G.; Lemaire, M. T.; Stevens, C. L. Journal of Organic Chemistry 1999, 64, 8893.

(2) Parks, J. E.; Wagner, B. E.; Holm, R. H. Journal of Organometallic Chemistry 1973, 56, 53.

(3) Cai, D. W.; Hughes, D. L.; Verhoeven, T. R. Tetrahedron Letters 1996, 37, 2537.

(4) Parks, J. E.; Wagner, B. E.; Holm, R. H. Inorganic Chemistry 1971, 10, 2472.

(5) Mukkala, V.-M.; Sund, C.; Kwiatkowski, M.; Pasanen, P.; Hogberg, M.; Kankare, J.;

Takalo, H. Helvetica Chimica Acta 1992, 75, 1621.

(6) Loren, J. C.; Siegel, J. S. Angewandte Chemie, Internation Edition English 2001, 40, 754.

(7) Stroh, C.; Ziessel, R. Tetrahedron Letters 1999, 40, 4543.

(8) El-Ghayouy, A.; Harriman, A.; De Cian, A.; Fischer, J.; Ziessel, R. Journal of the American Chemical Society 1998, 120, 9973.

(9) El-ghayoury, A.; Ziessel, R. Tetrahedron Letters 1998, 39, 4473.

(10) El-ghayoury, A.; Ziessel, R. Journal of Organic Chemistry 2000, 65, 7757. 